\title{
A novel origin for granulovacuolar degeneration in aging and Alzheimer's disease: parallels to stress granules
}

\author{
Rudy J Castellani ${ }^{1, *}$, Yashi Gupta ${ }^{2, *}$, Baiyang Sheng ${ }^{2}$, Sandra L Siedlak ${ }^{2}$, Peggy LR Harris ${ }^{2}$, Jeff M Coller ${ }^{3}$, \\ George Perry ${ }^{2,4}$, Hyoung-gon Lee², Massimo Tabaton ${ }^{5}$, Mark A Smith², Xinglong Wang ${ }^{2}$ and Xiongwei Zhu ${ }^{2}$
}

The phosphorylated ribosomal protein $\mathrm{S} 6$ (pS6) is associated with the $40 \mathrm{~S}$ ribosomal subunit in eukaryotes and is thought to have a role in RNA storage, degradation, and re-entry into translation. In this study, we found pS6 localized to granulovacuolar degeneration (GVD) within the pyramidal neurons. Immunohistochemical analysis found that nearly 20-fold more neurons contain pS6-positive granules in Alzheimer's disease (AD) hippocampus compared with agematched controls. Further, pS6-positive granules were more common in neurons not containing neurofibrillary tangles (NFTs), were never associated with extracellular NFTs or in apoptotic neurons, and contained less RNA than neighboring pyramidal neurons not containing pS6-positive granules. In model systems, pS6 is a specific marker for stress granules, and another stress granule protein, p54/Rck, was also found to be a component of GVD in the current study. Stress granules are transient, intracellular, dense aggregations of proteins and RNAs that accumulate as a stress response, protecting cells from apoptosis and inappropriate transcriptional activity, often described as a form of 'molecular triage.' The RNA oxidation modification 8-hydroxyguanosine (8OHG) is strikingly increased in AD, yet this study reports that those neurons with pS6 granules display reduced RNA oxidation demonstrated by lower levels of 8OHG. Since chronic oxidative stress is central to AD pathogenesis, and RNA is a specific oxidative stress target and is intimately associated with stress granule biogenesis in model systems, we suggest that GVD in human brain parallel stress granules, and may in fact be more representative of early disease pathogenesis than traditionally believed. This proposed origin for GVD as a neuroprotective response, may represent a morphologic checkpoint between cell death and reversible cellular stress that proceeds in the absence of other inclusions.

Laboratory Investigation (2011) 91, 1777-1786; doi:10.1038/labinvest.2011.149; published online 3 October 2011

KEYWORDS: Alzheimer's disease; granulovacuolar degeneration; ribosomal protein pS6; stress granules

Alzheimer's disease $(\mathrm{AD})$ is a slowly progressive neurodegenerative disease characterized by cognitive decline and accumulation of 'hallmark' lesions within the brain. $\mathrm{AD}$ is a strictly age-associated condition with a dramatic increase in incidence after age 65; approximately one-half of all individuals over the age of 85 are affected by AD. It is further estimated that $\sim 25$ million people are affected worldwide, and that by 2050, the prevalence in the United States alone is projected to be 16 million. ${ }^{1-3}$
The two classic hallmark lesions that occur within the brain are the neurofibrillary tangle (NFT) comprised of phosphorylated- $\tau$, and the senile plaque, comprised of amyloid- $\beta$. Noteworthy is that both lesions accumulate with age, generally to a lesser extent compared with $\mathrm{AD}$, although it is well known that substantial pathology, amyloid plaques in particular, often occurs in the elderly in the absence of clinical dementia. NFTs on the other hand show a more predictable progression over the course of the disease from

\footnotetext{
${ }^{1}$ Deparment of Pathology, University of Maryland, Baltimore, MD, USA; ${ }^{2}$ Department of Pathology, Case Western Reserve University, Cleveland, OH, USA;

${ }^{3}$ Center for RNA Molecular Biology, Case Western Reserve University, Cleveland, OH, USA; ${ }^{4}$ Department of Biology, UTSA Neurosciences Institute,

University of Texas at San Antonio, San Antonio, TX, USA and ${ }^{5}$ Department of Internal Medicine, University of Genoa, Genoa, Italy

Correspondence: Dr RJ Castellani, MD, Department of Pathology, University of Maryland, 22 South Greene Street, Baltimore, MD 21201, USA or Dr X Zhu, PhD,

Department of Pathology, Case Western Reserve University, 2103 Cornell Road, Cleveland, OH 44106, USA.

E-mails: rcastellani@som.umaryland.edu or xiongwei.zhu@case.edu

*These authors contributed equally to this work. 
medial temporal lobe regions early (entorhinal cortex, Sommer's sector, transentorhinal region, amygdala) to association cortex late, and to primary cortical areas by end stage. ${ }^{4}$ It is of further interest that given the direct role of the medial temporal lobe regions and the hippocampus in particular in processing of declarative memory, as well as its early involvement by NFTs in $\mathrm{AD}$, the hippocampus is viewed as a critical area in the disease process. Not surprisingly, the hippocampus is the most widely studied brain region in both humans and animals.

Also, predominantly in the hippocampus, granulovacuolar degeneration (GVD) is an invariable accompaniment of $\mathrm{AD}$. GVD is generally excluded from case assessment using standardized pathological criteria for disease diagnosis, due to its frequent presence as an incidental finding in controls (a quality shared with both senile plaques and NFTs), and its subtle appearance on microscopic examination, hampering quantitation and requiring high-magnification light microscopy to fully appreciate. GVD is not limited to medial temporal lobe, but may also be encountered in neocortical areas, deep gray matter structures including nucleus basalis, hypothalamus, and brainstem, as well as olfactory bulb. ${ }^{5}$

GVD is characterized morphologically by the presence of discrete $0.5-5 \mu \mathrm{m}$ basophilic granules, each within a clear vacuole, in the perikaryal cytoplasm of pyramidal neurons. The granules contain an electron dense core surrounded by a translucent matrix on ultrastructural analysis, and have been shown to have epitopes for neurofilament protein, phosphorylated- $\tau$, PIN1, ubiquitin, and, interestingly, mitotic proteins TG-3, and MPM-2. ${ }^{6-9}$ The myriad of proteins described in GVD represent the autophagic ubiquitinproteasome system, unfolded protein response, endosomal and lysosomal proteins, $\tau$ and amyloid processing events, and stress activated and mitogen-activated protein induction. ${ }^{8,10-15}$ In a variety of organisms, intracellular bodies referred to as stress granules, contain poly(A) mRNA, 40S ribosomal subunits, eIF4E, eIF4G, eIF4A, eIF4B, poly(A)binding protein, eIF3, and eIF2, and p54/Rck protein. ${ }^{16-18}$ They form following acute cellular stress of various etiologies (eg, heat shock, oxidative stress, viral infection, ischemia), when translational arrest ensues ${ }^{19}$ and proteins normally involved in mRNA processing assume 'emergency ancillary functions' activating a process of molecular triage as mRNAs from disassembled polysomes are sorted and their individual fate determined. This triage process takes place in the stress granule, indicating that stress granules are active participants in cellular metabolism associated with stress. The stress granule, as well as a related structure known as the processing body (P-body), also contain RNA-induced silencing complexes, suggesting that these RNA granules are integrated with microRNA-induced translational silencing pathways, thus affecting diverse cellular pathways and cell fate decisions.

It is also of interest that phosphorylation of the stress granule protein eIF2a is said to be activated by oxidative stress. ${ }^{19}$ Neuronal oxidative stress also triggers ERK activation, another protein readily detected in GVD, that in turn can induce S6 phosphorylation via induction of p70S6 kinase. ${ }^{20-22}$ We have previously demonstrated that sublethal oxidative damage of RNA occurs in $\mathrm{AD}^{23,24}$ In this study, we examined AD brains for the GVD presence of phosphorylated-protein S6 (pS6) protein, the absence of which is associated with decreased cell survival. ${ }^{25}$ Concomitant analyses for oxidative stress, neurofibrillary pathology, extracellular tangles, rRNA, and RNA oxidation and apoptosis were performed in an attempt to further characterize the mechanism of GVD formation. These findings, together with the morphological similarities between stress granules and GVD, provide a novel link between oxidative damage, RNA metabolism, mechanism of GVD formation, and neurodegeneration in $\mathrm{AD}$.

\section{MATERIALS AND METHODS Immunohistochemistry}

Samples of hippocampus were obtained at autopsy from the Brain Bank at Case Western Reserve University following approved IRB protocols. Samples were fixed in buffered formalin or methacarn (methanol:chloroform:acetic acid; 6:3:1), and embedded in paraffin and sectioned at $6 \mu \mathrm{m}$. In all, 13 cases of histopathologically diagnosed AD ('definite' $\mathrm{AD}$ by CERAD criteria, ${ }^{26}$ Braak Stage $\mathrm{V}-\mathrm{VI},{ }^{4}$ and 'high likelihood AD' by NIA-Reagan criteria ${ }^{27}$ were used, ranging from 67 to 89, mean 77.7 years, with an average postmortem interval of $11.3 \mathrm{~h} .12$ control cases with no history of neurologic disease during life were used, and included four younger cases (ages 19-42 years) and eight age-matched controls (ages 66-101 years, mean 78.9 years, mean postmortem interval of $18.8 \mathrm{~h}$ ). Similar numbers of female and male patients were examined in each group. Paraffinembedded tissue sections were rehydrated by placing them in two changes of xylene for $10 \mathrm{~min}$ each, followed by 10 -min treatments of $100,95,70,50 \%$ ethanol, and finally to tris-buffered saline $(50 \mathrm{mM}$ Tris, $150 \mathrm{mM} \mathrm{NaCl}, \mathrm{pH}=7.6)$. A 30-min incubation in $3 \% \mathrm{H}_{2} \mathrm{O}_{2}$ in methanol to remove endogenous peroxidase was performed after $95 \%$ ethanol. Sections were rinsed in TBS for $10 \mathrm{~min}$ before blocking in $10 \%$ normal goat serum in TBS for $30 \mathrm{~min}$ before applying primary antibodies. Antibodies used in this study are rabbit antibody against phospho-pS6 ribosomal protein phosphorylated at Ser 235/236 (pS6) (Cell Signaling 91B2), sheep phospho-pS6 ribosomal protein phosphorylated at Ser 235/ 236 (Abcam), mouse monoclonal to total S6 protein (Cell Signaling 54D2), rabbit antibody to p54/RCK (Bethyl Laboratories A300-460), Alz-50 (gift of Peter Davies), PHF1 (gift of S Greenberg), and AT8 (Pierce Endogen), monoclonal anti-8-hydroxyguanosine (8OHG; QED Biosciences), and anti-rRNA (Santa Cruz Biotechnology). The peroxidase-anti-peroxidase method ${ }^{28}$ was used and developed using DAB (Dako). For some experiments, double stain immunocytochemistry was performed using Fast Blue as chromagen. 


\section{Dephosphorylation}

For some experiments, adjacent sections were pretreated overnight with $5 \mathrm{U} / 100 \mu \mathrm{l}$ alkaline phosphatase (Sigma) in 0.1 M PMSF and $0.1 \mathrm{M}$ Tris $\mathrm{pH} 8.0$ before immunostaining with pS6 to demonstrate the specificity of the antibody for the phosphorylated form of pS6.

\section{Double Label Fluorescence Microscopy}

To directly compare some antibodies in the same intracellular granules, antibodies of different species were incubated together and detected with either Alexafluor 488 or Alexafluor 568 and imaged with a Zeiss Axiocam.

\section{TUNEL Assay}

Paraffin sections from three AD cases were stained for pS6 and developed using DAB. A $15-\mathrm{min} 10 \mu \mathrm{g} / \mathrm{ml}$ proteinase $\mathrm{K}$ treatment at $37^{\circ} \mathrm{C}$ was performed and the TUNEL assay was completed following the manufacturer's instructions (Roche). To visualize the TUNEL reaction at the light level, monoclonal antibody to FITC (Sigma) was used as a label at 1:500 and Fast Blue used as detection chromagen.

\section{Adsorption}

To further confirm the specificity of the antiserum, diluted pS6 ribosomal antibody was incubated with $20 \mu \mathrm{l}$ of its specific antigen peptide (Cell Signaling). After incubation for $16 \mathrm{~h}$, the adsorbed antibody was applied on serial sections next to unadsorbed antibody.

\section{bFGF Binding}

The bFGF-binding assay ${ }^{29}$ is used to demonstrate specifically extracellular NFTs. Three AD cases were stained with either pS6 alone, double stained for both pS6 and FGF, or bFGF alone. pS6 antibody was visualized with DAB before application of $0.5 \mu \mathrm{g} / \mathrm{ml}$ of hbFGF in $150 \mathrm{mM} \mathrm{NaCl}, 20 \mathrm{mM}$ EDTA, and $50 \mathrm{mM}$ Tris- $\mathrm{HCl}$ at $\mathrm{pH} 7.6$ at $37^{\circ} \mathrm{C}$ for $2 \mathrm{~h}$. Monoclonal antibody to bFGF was used to detect bound FGF and was detected using Fast Blue.

\section{Oxidative Stress}

A proteinase $\mathrm{K}$ pretreatment of $20 \mu \mathrm{g} / \mathrm{ml}$ for $15 \mathrm{~min}$ at $37^{\circ} \mathrm{C}$ was performed. Serial sections were then immunostained for $8 \mathrm{OHG}$ (clone 15A3, QED Biosciences) and pS6, and immunoreactions developed with DAB.

\section{Comparison with RNA Levels}

Adjacent serial sections from three cases of $\mathrm{AD}$ were stained with pS6 antibody and anti-rRNA. Images were taken of the same fields and the immunoreaction intensity of rRNA was measured in pyramidal neurons containing pS6 granules and in cells without pS6. Treatment of the sections with RNAse before application of primary antibody abolished all immunoreactivity of the rRNA antibody (data not shown).

\section{Quantification}

The number of pyramidal neurons containing pS6-positive granules in the entire hippocampus cornus ammonis regions CA1-CA4 at the level of the lateral geniculate nucleus were counted in one representative section from all AD and control cases. For some experiments, the pS6-positive inclusions were correlated with NFT pathology, in the sections double stained for phosphorylated- $\tau$ and pS6. Number of pS6positive cells in the entire CA1-CA4 regions was counted as either being positive for pS6 only, or positive for both pS6 and $\tau$. In all, $13 \mathrm{AD}$ cases and 12 control cases were analyzed.

\section{Densitometric Analysis}

Computer-assisted image analysis was used to measure the level of immunoreaction in 150-200 pyramidal neurons from three fields from the CA1/2 region of the hippocampus in the $\mathrm{AD}$ and control sections for the immunoreactions intensity of rRNA and 8OHG. Using a Zeiss axiocam and associated Axiovision software, adjacent fields with many of the same neurons present in both sections stained for phosphorylated pS6 and either rRNA or $8 \mathrm{OHG}$ were imaged. The pyramidal neurons were detected and the intensity of immunoreaction with the background level of the surrounding neuropil subtracted. The measurements for the neurons containing pS6 granules were separated from those values from neurons devoid of pS6. The average neuronal staining level for these two populations was determined for each case and the Student's $t$-test was used to statistically compare the different groups.

\section{RESULTS}

In the $\mathrm{AD}$ cases, strong immunoreactivity for the pS6 ribosomal protein was found in pyramidal neurons in the cornus ammonis regions of the hippocampus, the subiculum, and the parahippocampal gyrus. Specifically, small spherical structures within pyramidal neurons that corresponded morphologically to GVD were strongly positive (Figures $1 \mathrm{~b}$ and c). Young control individuals (ages 19, 27, 31, and 42 years old) showed no specific pS6 immunoreactivity (Figure 1a). When present in the older controls (ages over 66 years), the stained structures resembled incidental GVD. The number of neurons with pS6-positive granules was counted in the entire CA1, CA2, CA3, and CA4 regions of $\mathrm{AD}$ and control cases. This analysis revealed almost 20-fold more neurons, demonstrating pS6 staining in AD tissue compared with control (Figure 1d; $P<0.01$ ). Specifically, the hippocampus of the AD cases contained on average 195 ( \pm 41 s.e.m.) pS6-positive neurons and the control only 8 ( \pm 5 s.e.m.) pS6-positive neurons.

The antibody used is dependent on the phosphorylation of S235 and S236. To test this dependence, tissue sections were dephosphorylated with alkaline phosphatase before application of antibodies and all pS6-positive immunoreactivity was abolished (Figures 2a and b). Further, the specificity of the pS6 antibody was confirmed by adsorption with the pS6 

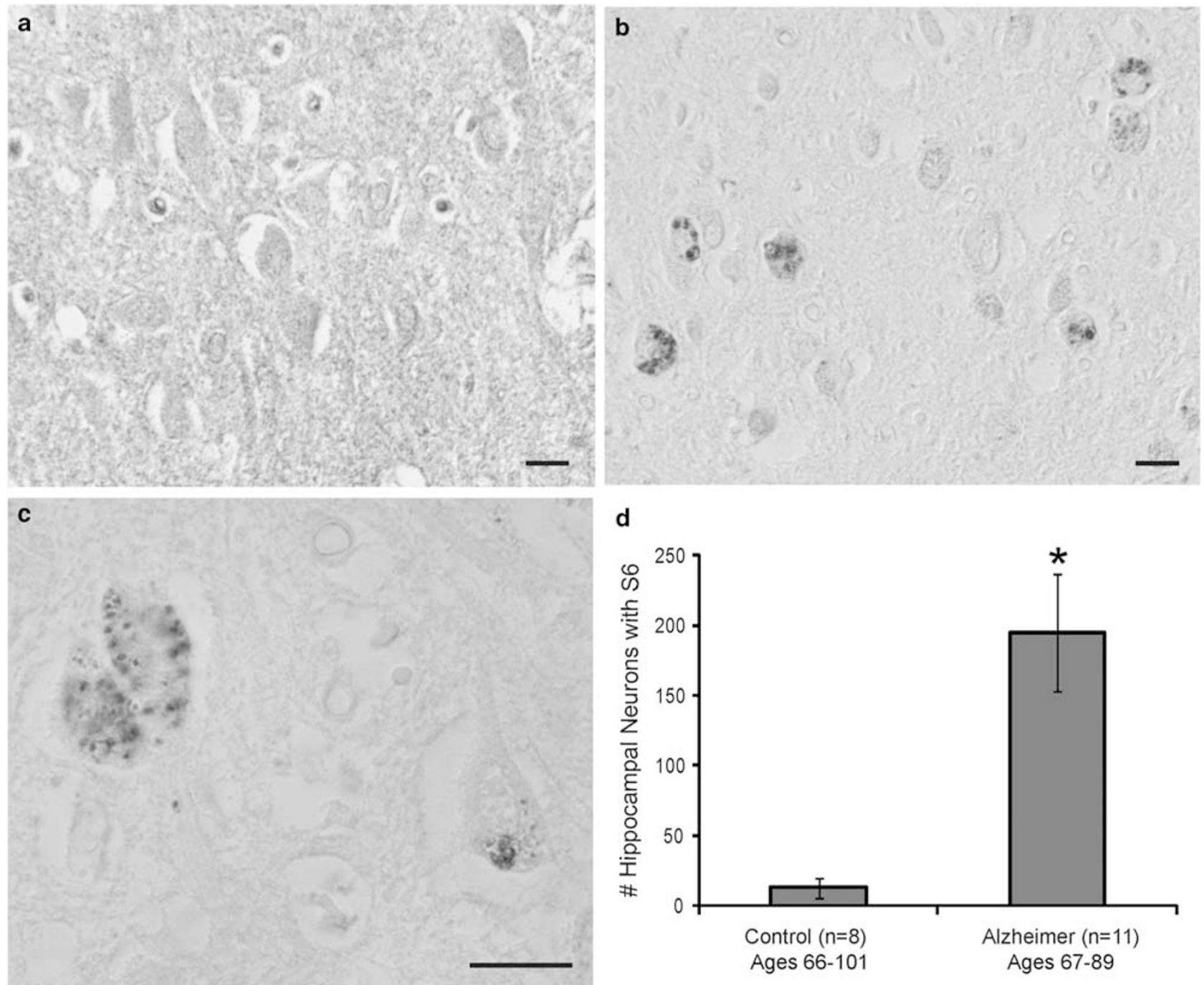

Figure 1 Hippocampus sections from control cases rarely display pS6 granular staining (a). In AD cases, however, many neurons have specific pS6 granules (b). Higher magnification reveals the granules range from about 0.1 to $2.0 \mu \mathrm{m}$ in diameter (c). Quantification of the number of neurons in the entire hippocampal section containing granules in the CA1, CA2, CA3, and CA4 regions, finds AD cases $(n=11)$ contain nearly 20 -fold more pS6 containing neurons than age-matched controls $(n=8)\left(\mathbf{d},{ }^{\star} P<0.01\right)$. Scale bars $=20 \mu \mathrm{m}$.

peptide, which eliminated all immunoreactivity (Figures $2 \mathrm{c}$ and d). An et al. ${ }^{30}$ previously demonstrated the specificity of this antibody in the human brain by immunoblot analysis.

To determine whether the granular staining can also be detected using a phosphorylation-independent S6 antibody, a mouse monoclonal recognizing total $\mathrm{S} 6$ protein was applied. In addition to the morphologically similar GVD structures, many pyramidal neurons in the $\mathrm{AD}$ cases demonstrated diffuse cytoplasmic reactivity (Figure 3e). Double label fluorescent microscopy revealed that many of the same structures are recognized by the antibodies to both total S6 and pS6 ribosomal protein (Figures 3a-c). To further confirm the specificity, a second polyclonal antibody to pS6, one raised in sheep, also specifically labeled the GVD/stress granule structures in the $\mathrm{AD}$ cases (Figure $3 \mathrm{~d}$ ). A protein that is specifically located to experimentally induced stress granules in model systems, p54/RCK, was also found to localize strikingly to the same structures (Figure 3f).

In the sections double stained for phosphorylated- $\tau$, using either Alz-50 or PHF1, and pS6, some colocalization was seen within neurons containing NFTs (Figures $4 \mathrm{a}$ and b). Counting the number of cells within the entire hippocampus of four AD cases containing either pS6 only, or both pS6 and phosphorylated- $\tau$, revealed that NFT are present in $40 \pm 17 \%$ of those cells with pS6-positive granules (Figure 4c).

To further characterize the neuronal populations that demonstrate pS6-positive granules, DNA fragment labeling technique, TUNEL, was performed to study the relationship of pS6 granules in the apoptotic signaling cascade of hippocampal neurons. Analysis of three AD cases found 

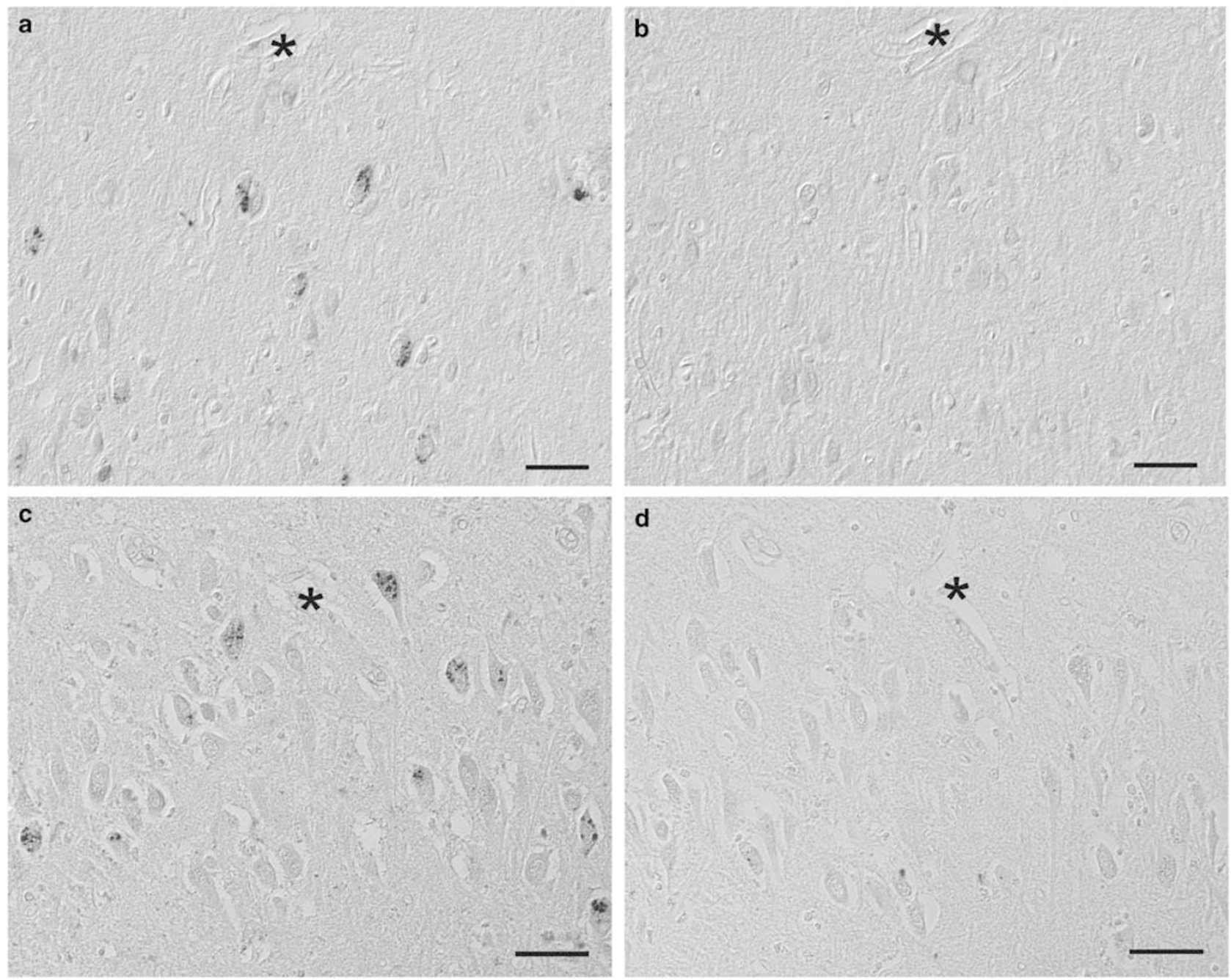

Figure 2 Compared with an untreated section of AD hippocampus (a), dephosphorylation of an adjacent tissue section completely abolished all immunoreactivity for pS6 (b). Adsorption with pS6 peptide (d) also abolished all granular staining as seen on adjacent section (c). ${ }^{\star}$ denotes landmark vessels in (a-d). Scale bars $=50 \mu \mathrm{m}$.

pyramidal neurons with TUNEL-positive nuclei never colocalized with neurons with pS6-positive granules (Figure 5a). Double staining experiments on three AD cases comparing pS6 and the FGF-binding assay, which specifically labels extracellular NFTs, also showed no overlap (Figure 5b).

Since the accumulation of GVD within AD neurons has been associated with a decrease in RNA levels, ${ }^{6}$ we sought to examine whether the appearance of the pS6-positive granules correlate with decreased levels of RNA within individual neurons. Serial adjacent sections were immunostained for pS6 protein and with an antibody against ribosomal RNA (Figures $6 a$ and $b$ ). From three AD cases, images of the same adjacent fields were obtained with an Axiocam using a $\times 20$ lens on the Axiophot. Computer-assisted densitometric analysis was used to measure the cytoplasmic intensity of the rRNA immunoreactions in all pyramidal neurons from three fields of the CA1 and CA2 regions. The measurements were separated by whether the neurons contained pS6-positive granules, as determined from the adjacent sections stained for pS6. Quantification of the staining of adjacent sections with pS6 and rRNA found that those cells containing pS6 granules have significantly lower levels of rRNA (on average $20 \%$ less) than neighboring pyramidal neurons without pS6 $(P<0.01)$ (Figure $6 \mathrm{c})$. The same experiment was performed comparing neuronal levels of $8 \mathrm{OHG}$ and pS6. Examination of the adjacent sections found that those neurons with pS6 granules also had significantly lower levels of cytoplasmic $8 \mathrm{OHG}$ (on average $50 \%$ less) (Figure $7 ; P<0.01$ ).

\section{DISCUSSION}

The appearance of stress granules has been associated with a variety of cellular stressors, including oxidative stress, that induces phosphorylation of the eukaryotic initiation factor eIF2a and is a site for the accumulation of the phosphorylated ribosomal S6 protein (pS6). ${ }^{19}$ In this study, we identified pS6 granules in $\mathrm{AD}$ hippocampus and found it 

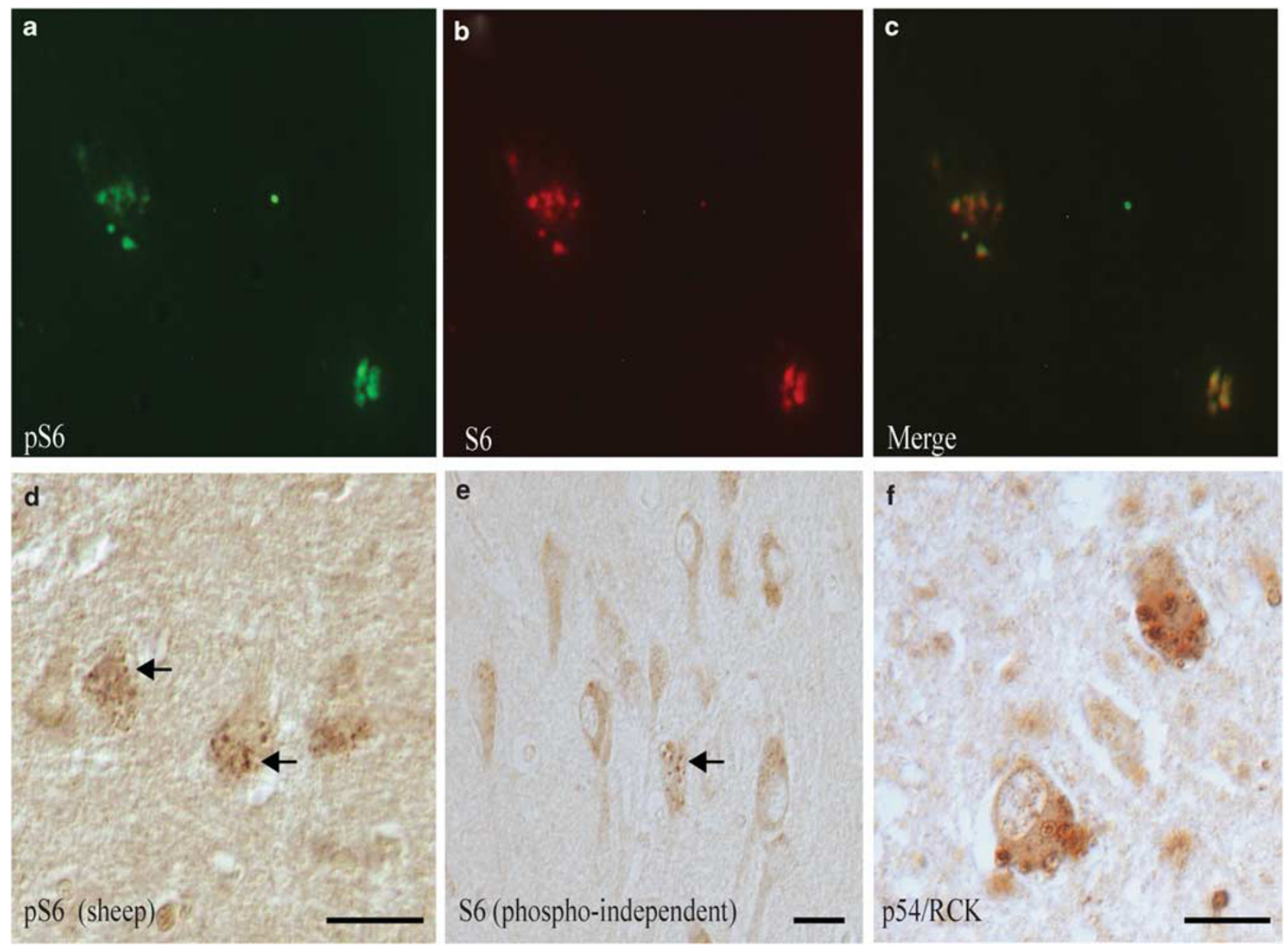

Figure 3 The granular staining can also be detected using a mouse monoclonal phosphorylation-independent S6 antibody, recognizing total S6 protein (e). In addition to the morphologically similar GVD structures (arrow), many pyramidal neurons in the AD cases demonstrated diffuse cytoplasmic reactivity. Double label fluorescent microscopy revealed many of the same structures are recognized by the antibodies to both pS6 (a) and total S6 (b) and (c, merged image). A polyclonal antibody to pS6 raised in sheep also specifically labels the GVD/stress granule structures in the AD cases (d, arrows). p54/RCK was also found to localize strikingly to the same structures (f). Scale bars $=25 \mu \mathrm{m}$.

specifically localized to intracellular structures morphologically similar to GVD and correlated these structures with other neurodegenerative processes. One hypothesis stemming from the data observed in this study is that a population of GVD may represent the chronic and thus more static pathological structure originated from stress granules, and as such represents a highly conserved marker of cellular stress. The fact that phosphorylated S6 was associated with decreased 8OHG but never associated with TUNEL or extracellular NFTs suggests that GVD, similar to stress granule formation, may represent an adaptive mechanism that leads to neuroprotection. Moreover, whereas GVD is detectable in $\mathrm{AD}$ as well as aged controls, and is not used in standardized diagnostic criteria, this study suggests that GVD may be a more important marker of aging and neurodegeneration than previously believed. Given the current emphasis on functional and preclinical changes over structural and overt clinical disease for therapeutic intervention, the GVD/stress granule response offers an attractive target as an early change. Moreover, its significance in terms of early clinical disease has not been systematically examined to date.

Stress granule formation appears to have a protective role since impaired stress granule formation often leads to increased cell death. ${ }^{25}$ In parallel with these studies, we found neuronal pS6 granule formation in AD brain is neither correlated with TUNEL colocalization, indicating that the apoptotic pathway is separate from the pS6 granule formation, nor associated with extracellular NFT. ${ }^{31}$ The cytoplasmic GVD-associated labeling for pS6 protein along with the negative association with apoptosis is intriguing, in light of studies showing potential for nuclear translocation of phosphorylated pS6, transcription activation, cell-cycle activation, and induction of apoptosis. ${ }^{25}$ Sequestration of apoptotic regulators in stress granules limits downstream apoptotic signaling. ${ }^{32}$ Along this line, it is of interest to note that some studies have found activated caspase- 3 localized to 

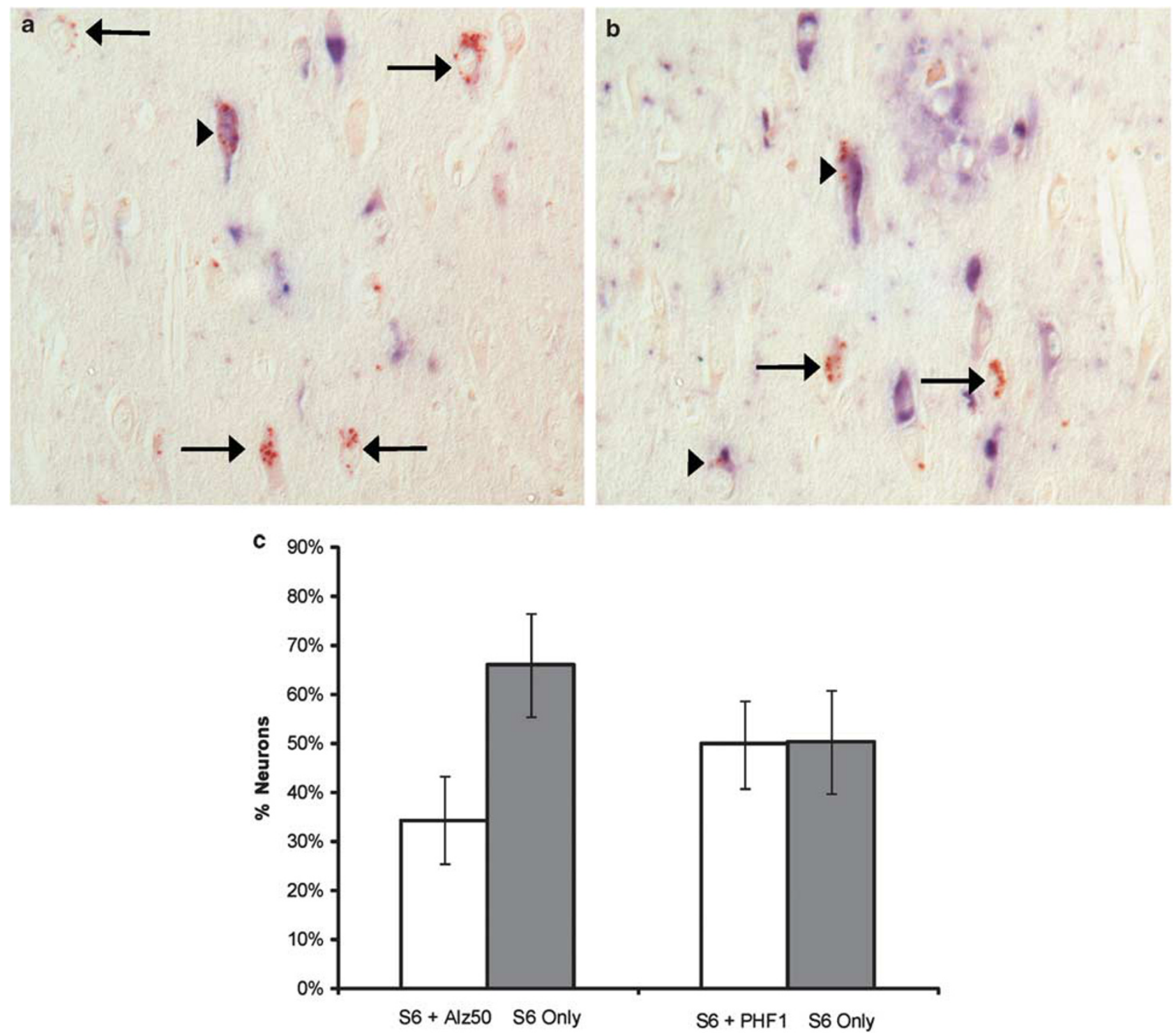

Figure 4 Double staining of pS6 (brown) with either Alz-50 (blue, a) or PHF1 (blue, b) of AD hippocampus. In both (a, b), arrows mark neurons with no colocalization while arrowheads indicate colocalization with $\tau$. Quantification of the entire CA1, CA2, CA3, and CA4 regions from four AD cases reveals that $\tau$ is present in $\sim 40 \pm 17 \%$ of those cells with pS6-positive granules (c).

a subset of $\mathrm{GVD}^{33}$ in cells that lack other morphological characteristics of apoptosis, suggesting that sequestration of caspase-3 into subcellular compartment such as GVD acts to protect the neuron. Taken together, this suggests that GVD may represent the cellular response to a sublethal cellular insult, or an adaptive response to the neurodegenerative process and that, in analogy to stress granules in model systems, a threshold exists beyond which deleterious cell-cycle activation and cell death occurs. GVD may thus be an important marker of a critical and reversible pivot point between cell death and cell survival that may serve as a useful target for therapeutic intervention. This construct is further consistent with our previous studies, indicating that cell-cycle activation and abortive apoptosis are intimately associated with neurodegeneration. ${ }^{34-36}$
Classical studies documented that RNA levels are decreased in AD. After staining freshly fixed biopsy samples with Azure B, RNA content was measured in the cytoplasm, nucleus, and nucleolus, and revealed significant loss of RNA in neurons from cases diagnosed with $\mathrm{AD}$ also. ${ }^{6,37}$ Further studies corroborated the finding of reduced RNA levels, and found that this reduction correlated with increased ribonuclease activity. ${ }^{38}$ However, it is unclear whether reduced RNA content represents a detrimental event during the degenerative cascade or an adaptive response. In this regard, given that pyramidal neurons containing pS6 granules are never associated with apoptotic markers, our finding that there is significantly less RNA observed in these cells with pS6 granules is of significance to suggest that RNA reduction may represent a neuroprotective response. Stress granules are 

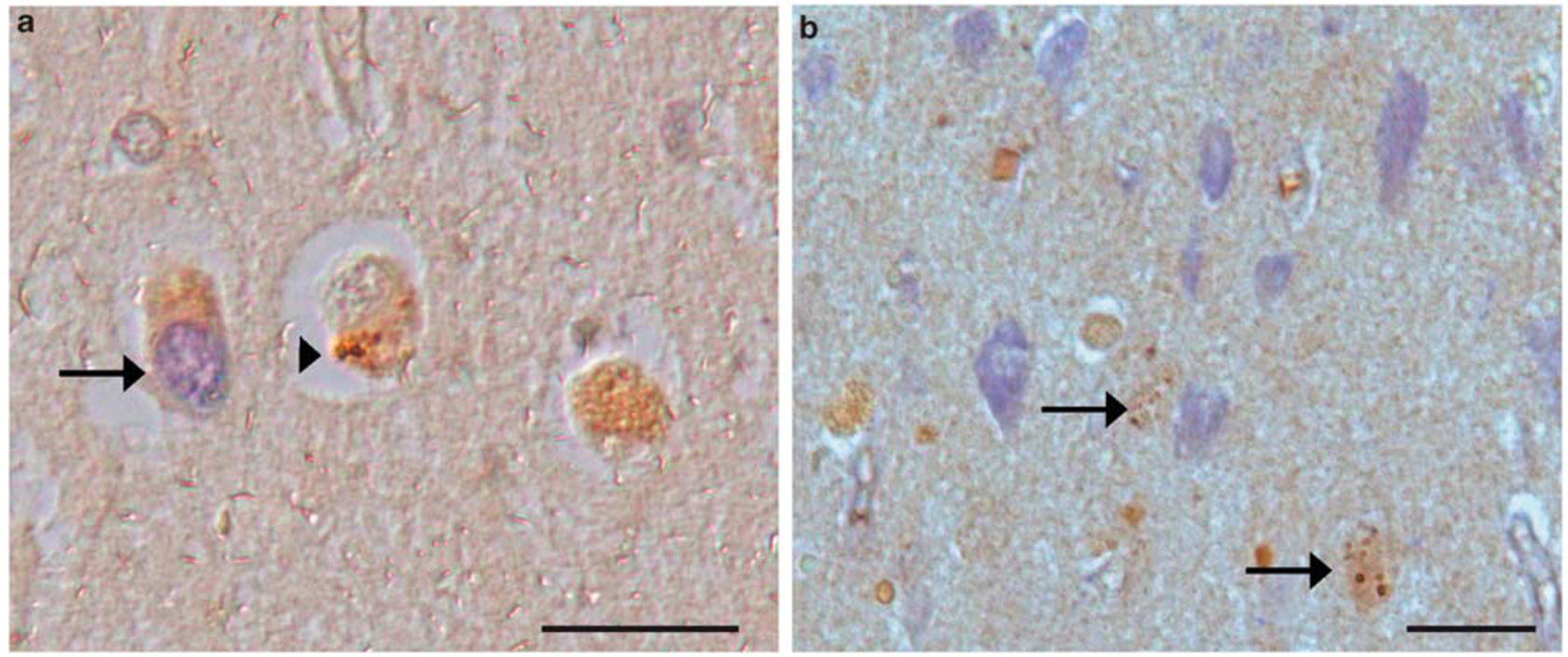

Figure 5 In AD hippocampal tissue sections, neurons with TUNEL-positive nuclei (blue, arrow) show no colocalization with pS6 granules (brown, arrowhead) (a). No colocalization of pS6 granules (brown, arrows) is observed with the bFGF assay, which stains mostly extracellular NFT (blue) (b). Scale bars $=50 \mu \mathrm{m}$.

thought to serve as a protective and decision mechanism for RNAs. ${ }^{18}$ As an acute response, the formation of stress granules temporarily represses global translation and also preserves mRNA. ${ }^{18}$ It is not uncommon for cells to employ additional mechanisms such as the cleavage of $\mathrm{RNA}^{39}$ and/or decrease in $\mathrm{rRNA}^{40}$ to ensure translational repression. Along this line of reasoning, in $\mathrm{AD}$, it is possible that reduced rRNA levels may represent an adaptive response in addition to the $\mathrm{GVD} /$ stress granule formation to ensure translation repression during the chronic stress neurons are facing and that those neurons with such successful adaptations have a larger chance to survive.

In previous studies, we uncovered an important deleterious consequence of oxidative injury to RNA. ${ }^{23}$ Because RNA is largely single stranded and its bases lack the protection of hydrogen bonding and binding by specific proteins, RNA may be more susceptible to oxidative insults than DNA. Oxidative damage to protein-coding RNA or non-coding RNA will, in turn, potentially cause errors in proteins and/or dysregulation of gene expression. While less lethal than mutations in the genome, such sublethal insults to cells may be associated with underlying mechanisms of several chronic diseases, including $\mathrm{AD}^{41}$ Increased RNA oxidation is indeed a striking characteristic of the susceptible neuronal population in $\mathrm{AD} .{ }^{23,42,43}$ We found that the majority of cells with granules have significantly less oxidized RNA, displaying the modification $8 \mathrm{OHG}$, in the cytoplasm. Such a great reduction of oxidized RNA cannot be solely explained by modest decrease in the rRNA levels in these cells, and we suspect that GVD/stress granule formation, as an adaptive response, may reduce RNA oxidation through either degradation or repair of damaged RNA. It is not unexpected that the GVD do not contain $8 \mathrm{OHG}$, as only undamaged RNAs would be protected while damaged ones would be selectively degraded. Although it is not clear what specific stressor(s) induce $\mathrm{GVD} /$ stress granule formation in $\mathrm{AD}$ neurons, the abundance of $8 \mathrm{OHG}$ and oxidative stress in $\mathrm{AD}$ brain along with the differential display of $8 \mathrm{OHG}$ levels between neurons with and without GVD suggest that it is likely the chronic oxidative stress and more specifically, RNA oxidation contributes to GVD induction.

In agreement with previous studies, there is a lack of correlation between GVD and neruofibrillary pathology. For instance, Pin 1, CK1, SAPK all show variable colocalization with NFT. ${ }^{13,44}$ Many studies also report that as GVD numbers increase, NFT levels also increase within the same brain region, evidence for GVD being a specific AD-related change. Along these lines, not surprisingly, we found a small number of neurons containing phosphorylated pS6 in control cases, and then only in older controls. GVD is a known morphological change in the hippocampus of aged individuals with or without $\mathrm{AD}$, although they tend to be more numerous in $\mathrm{AD}^{45}$ However, our study provided even stronger evidence by comparing pS6-positive granules and phosphorylated- $\tau$ within the same neurons. Less than half of all pS6-positive cells develop NFT. Further, since neither extracellular NFT nor apoptotic cells contain these GVD, these granulovacuolar bodies may represent an early, or intriguingly, a reversible and protective structure. GVD may be a misnomer as their formation may not lead to, and may in fact protect neurons from, degenerating.

In summary, this is the first study that establishes a molecular link, namely the presence of phosphorylated ribosomal protein pS6, between stress granules and the long known change of GVD in the human brain. In effect, by both morphology and molecular signatures, GVD is the chronic and more static pathological structure originated from stress granules, and as such is due to RNA oxidation whose 

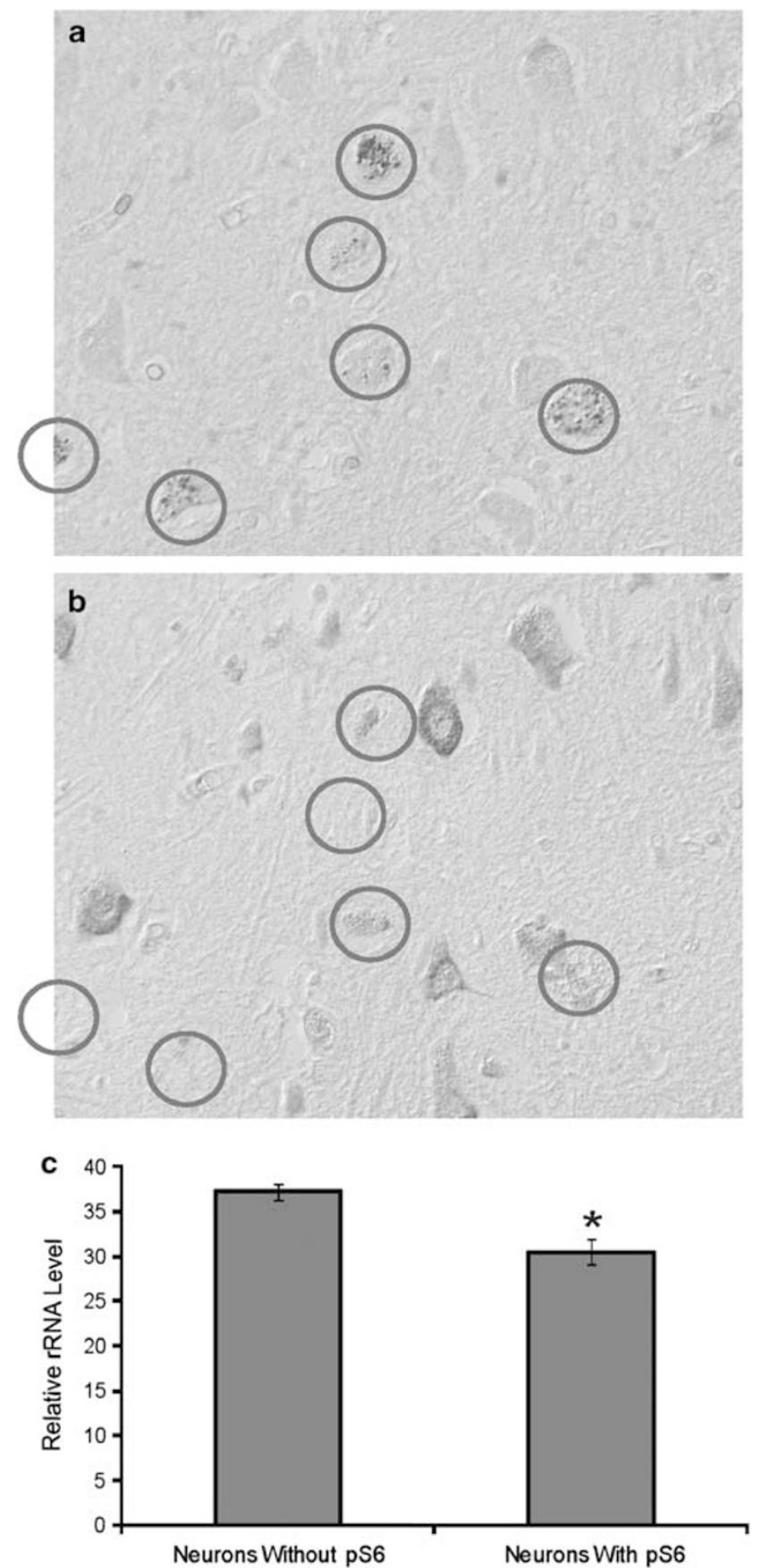

Figure 6 Adjacent serial sections of AD hippocampus stained with pS6 (a) and rRNA (b). Following quantification of the cytoplasmic levels of rRNA, cells with pS6-positive granules (circles) contain significantly less rRNA $\left({ }^{*}<<0.01\right)$ (c). The colour reproduction of this figure is available at the Laboratory Investigation journal online.

development represents a neuronal population that rarely ends with neuronal death. Our findings confirm the pathogenic link between RNA oxidation and aging/neurodegeneration, and indicate the importance of GVD and thus stress granules in neurodegenerative disease pathogenesis, and as a target for therapeutic intervention.
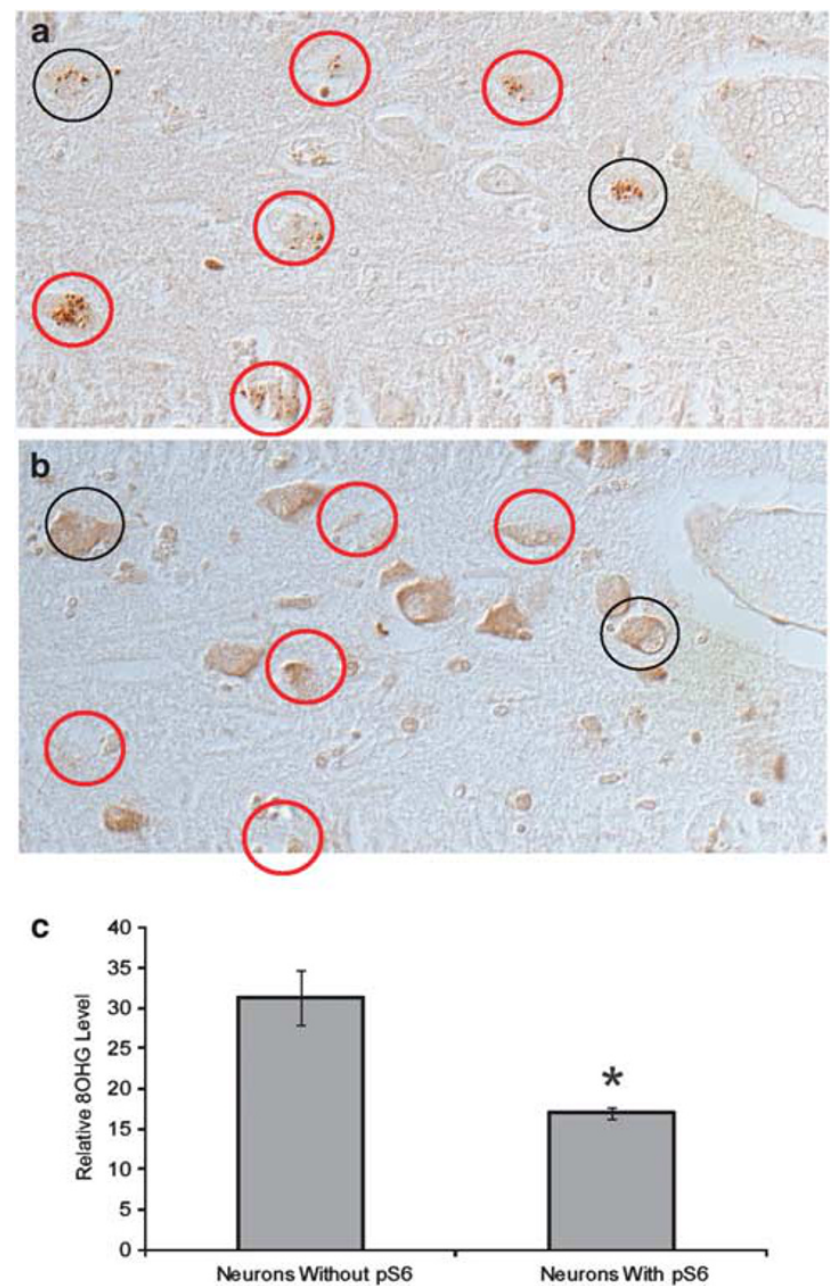

Figure 7 Adjacent serial sections of AD hippocampus stained for pS6 (a) and $8 \mathrm{OHG}$ (b). The majority of cells with pS6-positive granules contain visibly less $8 \mathrm{OHG}$ (red circles), while $\sim 25 \%$ of the pS6-positive neurons have $8 \mathrm{OHG}$ levels comparable to cells not containing pS6 (black circles). Following quantification of the cytoplasmic levels of $8 \mathrm{OHG}$, cells with pS6positive granules contain significantly less $8 \mathrm{OHG}\left(\mathbf{c},{ }^{*} P<0.01\right)$.

\section{ACKNOWLEDGEMENT}

The work in the authors' laboratories was supported by the National Institutes of Health (AG028679 to HGL; AG031852 to XWZ).

\section{DISCLOSURE/CONFLICT OF INTEREST}

Dr Perry is a consultant for Takeda Pharmaceuticals and Neurotez and owns stock options in Neurotez and Voyager. Dr Zhu was a consultant for and received grant support from Medivation. Dr Smith was a consultant for Anavex Life Sciences Corporation, Eisai, Medivation, Neurotez, and Takeda Pharmaceuticals; owned stock options in Aria Neurosciences, Neurotez, Panancea, and Voyager, and had received lecture fees from GSK, Medivation, and Pfizer.

1. Smith MA. Alzheimer disease. Int Rev Neurobiol 1998;42:1-54.

2. Ziegler-Graham K, Brookmeyer R, Johnson $\mathrm{E}$, et al. Worldwide variation in the doubling time of Alzheimer's disease incidence rates. Alzheimers Dement 2008:4:316-323.

3. Brookmeyer R, Johnson E, Ziegler-Graham $\mathrm{K}$, et al. Forecasting the global burden of Alzheimer's disease. Alzheimers Dement 2007;3:186-191. 
4. Braak H, Braak E. Neuropathological stageing of Alzheimer-related changes. Acta Neuropathol (Berl) 1991;82:239-259.

5. Ball MJ. Topographic distribution of neurofibrillary tangles and granulovacuolar degeneration in hippocampal cortex of aging and demented patients. A quantitative study. Acta Neuropathol 1978;42:73-80.

6. Mann DM. Granulovacuolar degeneration in pyramidal cells of the hippocampus. Acta Neuropathol 1978;42:149-151.

7. Vincent I, Zheng JH, Dickson DW, et al. Mitotic phosphoepitopes precede paired helical filaments in Alzheimer's disease. Neurobiol Aging 1998;19:287-296.

8. Lagalwar S, Berry RW, Binder LI. Relation of hippocampal phospho-SAPK/ JNK granules in Alzheimer's disease and tauopathies to granulovacuolar degeneration bodies. Acta Neuropathol 2007;113:63-73.

9. Dakson A, Yokota O, Esiri M, et al. Granular expression of prolylpeptidyl isomerase PIN1 is a constant and specific feature of Alzheimer's disease pathology and is independent of tau, Abeta and TDP-43 pathology. Acta Neuropathol 2011;121:635-649.

10. Salminen A, Kauppinen A, Suuronen T, et al. ER stress in Alzheimer's disease: a novel neuronal trigger for inflammation and Alzheimer's pathology. J Neuroinflammation 2009;6:41.

11. Perry G, Roder $\mathrm{H}$, Nunomura $\mathrm{A}$, et al. Activation of neuronal extracellular receptor kinase (ERK) in Alzheimer disease links oxidative stress to abnormal phosphorylation. Neuroreport 1999;10:2411-2415.

12. Yamazaki $Y$, Takahashi T, Hiji M, et al. Immunopositivity for ESCRT-III subunit CHMP2B in granulovacuolar degeneration of neurons in the Alzheimer's disease hippocampus. Neurosci Lett 2010;477:86-90.

13. Kannanayakal TJ, Tao $\mathrm{H}$, Vandre DD, et al. Casein kinase- 1 isoforms differentially associate with neurofibrillary and granulovacuolar degeneration lesions. Acta Neuropathol 2006;111:413-421.

14. Funk KE, Mrak RE, Kuret J. Granulovacuolar degeneration (GVD) bodies of Alzheimer's disease (AD) resemble late-stage autophagic organelles. Neuropathol Appl Neurobiol 2011;37:295-306.

15. Hoozemans JJ, van Haastert ES, Nijholt DA, et al. The unfolded protein response is activated in pretangle neurons in Alzheimer's disease hippocampus. Am J Pathol 2009;174:1241-1251.

16. Wilczynska A, Aigueperse $C$, Kress $M$, et al. The translational regulator CPEB1 provides a link between dcp1 bodies and stress granules. J Cell Sci 2005;118(Pt 5):981-992.

17. Anderson $P$, Kedersha N. Stress granules: the Tao of RNA triage. Trends Biochem Sci 2008;33:141-150.

18. Buchan JR, Parker R. Eukaryotic stress granules: the ins and outs of translation. Mol Cell 2009;36:932-941.

19. Anderson P, Kedersha N. Stress granules. Curr Biol 2009;19:R397-R398.

20. Wang L, Gout I, Proud CG. Cross-talk between the ERK and p70 S6 kinase (S6K) signaling pathways. MEK-dependent activation of S6K2 in cardiomyocytes. J Biol Chem 2001;276:32670-32677.

21. Welsh GI, Hall DA, Warnes A, et al. Activation of microtubule-associated protein kinase (Erk) and p70 S6 kinase by D2 dopamine receptors. J Neurochem 1998;70:2139-2146.

22. Sarkar SN, Smith LT, Logan SM, et al. Estrogen-induced activation of extracellular signal-regulated kinase signaling triggers dendritic resident mRNA translation. Neuroscience 2010;170:1080-1085.

23. Nunomura A, Perry G, Pappolla MA, et al. RNA oxidation is a prominent feature of vulnerable neurons in Alzheimer's disease. J Neurosci 1999;19:1959-1964.

24. Honda K, Smith MA, Zhu X, et al. Ribosomal RNA in Alzheimer disease is oxidized by bound redox-active iron. J Biol Chem 2005;280: 20978-20986.

25. Eisinger-Mathason TS, Andrade J, Groehler AL, et al. Codependent functions of RSK2 and the apoptosis-promoting factor TIA-1 in stress granule assembly and cell survival. Mol Cell 2008;31:722-736.
26. Mirra SS, Heyman A, McKeel D, et al. The Consortium to Establish a Registry for Alzheimer's Disease (CERAD). Part II. Standardization of the neuropathologic assessment of Alzheimer's disease. Neurology 1991;41:479-486.

27. The National Institute on Aging, and Reagan Institute Working Group on Diagnostic Criteria for the Neuropathological Assessment of Alzheimer's Disease. Consensus recommendations for the postmortem diagnosis of Alzheimer's disease. Neurobiol Aging 1997;18 (4 Suppl):S1-S2.

28. Sternberger LA. Immunocytochemistry. New York: Wiley, 1986.

29. Siedlak SL, Cras P, Kawai M, et al. Basic fibroblast growth factor binding is a marker for extracellular neurofibrillary tangles in Alzheimer disease. J Histochem Cytochem 1991;39:899-904.

30. An WL, Cowburn RF, Li L, et al. Up-regulation of phosphorylated/ activated p70 S6 kinase and its relationship to neurofibrillary pathology in Alzheimer's disease. Am J Pathol 2003;163:591-607.

31. Cande C, Vahsen N, Metivier D, et al. Regulation of cytoplasmic stress granules by apoptosis-inducing factor. J Cell Sci 2004;117(Pt 19): 4461-4468.

32. Arimoto $\mathrm{K}$, Fukuda $\mathrm{H}$, Imajoh-Ohmi $\mathrm{S}$, et al. Formation of stress granules inhibits apoptosis by suppressing stress-responsive MAPK pathways. Nature Cell Biol 2008;10:1324-1332.

33. Stadelmann C, Deckwerth TL, Srinivasan A, et al. Activation of caspase3 in single neurons and autophagic granules of granulovacuola degeneration in Alzheimer's disease. Evidence for apoptotic cell death. Am J Pathol 1999;155:1459-1466.

34. Raina AK, Hochman A, Zhu X, et al. Abortive apoptosis in Alzheimer's disease. Acta Neuropathol (Berl) 2001;101:305-310.

35. McShea A, Harris PL, Webster KR, et al. Abnormal expression of the cell cycle regulators P16 and CDK4 in Alzheimer's disease. Am J Pathol 1997;150:1933-1939.

36. McShea A, Wahl AF, Smith MA. Re-entry into the cell cycle: a mechanism for neurodegeneration in Alzheimer disease. Med Hypotheses 1999;52:525-527.

37. Mann DM, Neary D, Yates PO, et al. Alterations in protein synthetic capability of nerve cells in Alzheimer's disease. J Neurol Neurosurg Psychiatry 1981;44:97-102.

38. Sajdel-Sulkowska EM, Marotta CA. Alzheimer's disease brain alterations in RNA levels and in a ribonuclease-inhibitor complex. Science 1984;225:947-949.

39. Thomas MG, Loschi M, Desbats MA, et al. RNA granules: the good, the bad and the ugly. Cell Signal 2011;23:324-334.

40. Vincent F, Corral-Debrinski M, Adolphe M. Transient mitochondrial transcript level decay in oxidative stressed chondrocytes. J Cell Physiol 1994;158:128-132.

41. Castellani RJ, Nunomura A, Rolston RK, et al. Sublethal RNA oxidation as a mechanism for neurodegenerative disease. Int J Mol Sci 2008; 9:789-806.

42. Nunomura A, Perry G, Aliev G, et al. Oxidative damage is the earliest event in Alzheimer disease. J Neuropathol Exp Neurol 2001;60: 759-767.

43. Ding Q, Markesbery WR, Cecarini V, et al. Decreased RNA, and increased RNA oxidation, in ribosomes from early Alzheimer's disease. Neurochem Res 2006;31:705-710.

44. Holzer M, Gartner U, Stobe A, et al. Inverse association of Pin1 and tau accumulation in Alzheimer's disease hippocampus. Acta Neuropathol 2002;104:471-481.

45. Dickson DW, Liu WK, Kress $\mathrm{Y}$, et al. Phosphorylated tau immunoreactivity of granulovacuolar bodies (GVB) of Alzheimer's disease: localization of two amino terminal tau epitopes in GVB. Acta Neuropathol 1993;85:463-470. 